\title{
Number of Lymph Nodes Positive for Tumor by IHC Staining Only
}

National Cancer Institute

\section{Source}

National Cancer Institute. Number of Lymph Nodes Positive for Tumor by IHC Staining

Only. NCI Thesaurus. Code C157145.

The quantity of lymph nodes that were positive for a neoplastic lesion with immunohistochemical (IHC) staining procedures. 